\title{
A new least-squares approach to differintegration modeling
}

\author{
Manuel D. Ortigueira ${ }^{\mathrm{a}, \mathrm{b}, *}$, António J. Serralheiro ${ }^{\mathrm{b}, \mathrm{c}}$ \\ ${ }^{a}$ UNINOVA and DEE, Faculdade de Ciências e Tecnologia da Universidade Nova de Lisboa, Campus da FCT da UNL, \\ Quinta da Torre, 2829-516 Caparica, Portugal \\ ${ }^{\mathrm{b}}$ INESC ID, Rua Alves Redol, 9, 2 , 1000-029 Lisboa, Portugal \\ ${ }^{\mathrm{c}}$ Academia Militar and CINAMIL, Rua Gomes Freire, 1150-175 Lisboa, Portugal
}

Received 20 May 2005; received in revised form 25 October 2005; accepted 14 December 2005

Available online 15 March 2006

\begin{abstract}
In this paper a new least-squares (LS) approach is used to model the discrete-time fractional differintegrator. This approach is based on a mismatch error between the required response and the one obtained by the difference equation defining the auto-regressive, moving-average (ARMA) model. In minimizing the error power we obtain a set of suitable normal equations that allow us to obtain the ARMA parameters. This new LS is then applied to the same examples as in [R.S. Barbosa, J.A. Tenreiro Machado, I.M. Ferreira, Least-squares design of digital fractional-order operators, FDA'2004 First IFAC Workshop on Fractional Differentiation and Its Applications, Bordeaux, France, July 19-21, 2004, P. Ostalczyk, Fundamental properties of the fractional-order discrete-time integrator, Signal Processing 83 (2003) 2367-2376] so performance comparisons can be drawn. Simulation results show that both magnitude frequency responses are essentially identical. Concerning the modeling stability, both algorithms present similar limitations, although for different ARMA model orders.
\end{abstract}

(C) 2006 Elsevier B.V. All rights reserved.

Keywords: Discrete-time fractional differintegrator; Pseudo-fractional ARMA; Grünwald-Letnikov derivative; Tustin bilinear transformation

\section{Introduction}

Fractional linear systems are described by fractional differential equations in the continuous-time case or ARMA models in the discrete-time case. The first case uses the definition of fractional derivative

\footnotetext{
*Corresponding author. UNINOVA and DEE, Faculdade de Ciências e Tecnologia da Universidade Nova de Lisboa, Campus da FCT da UNL, Quinta da Torre, 2829-516 Caparica, Portugal. Tel.: + 351212948520 ; fax: + 351212957786 .

E-mail addresses: mdortigueira@uninova.pt, mdo@dee.fct.unl.pt (M.D. Ortigueira), antonia.serralheiro@inesc-id.pt (A.J. Serralheiro).
}

[1]; the second uses the fractional differencing [2] The long memory exhibited by these systems cannot be explained by the usual integer order pole/zero models. The basic building block of this kind of systems is the non-integer order derivative that has been approximated by fractional powers of the backward difference or the bilinear transformations (the former is exactly the building block of the fractional differencing). These approximations are IIR systems with non-rational transfer functions. However, these more correct models are difficult to implement and model in practice. For them, ARMA models are only approximations that we 
call pseudo-fractional ARMA models [3]. In the last few years, a lot of attempts to obtain such models have been done (see [4-9]). However, it remains to clarify two important questions: (a) how to perform such modeling and (b) how to choose the most suitable orders. In impulse response modeling the well-known Padé algorithm is frequently used [2]. In [3], we presented a suitable recursive algorithm for this modeling. Here, we will propose a different algorithm based on a least-squares (LS) criterion different from [4]. This algorithm defines a mismatch error between the required response and the one obtained by the difference equation defining the ARMA model. In minimizing the error power we obtain suitable normal equations that allow us to obtain the ARMA parameters. The algorithm is described in Section 2 where we compare it with the algorithm described in [3] through some current modeling examples.

\section{Least-squares ARMA approximation}

\subsection{The discrete-time fractional differintegrator}

The differintegrator is a continuous-time linear system with transfer function given, in the causal case, by

$$
F(s)=s^{\alpha}
$$

for $\operatorname{Re}(s)>0$ [10]. To obtain discrete-time differintegrators, we replace the variable $s$ in (1) by a suitable rational function of $z^{-1}$. The most commonly used are:

(a) the backward difference, leading to a solution that is essentially the discretization of the Grünwald-Letnikov derivative;

(b) the Tustin bilinear transformation.

Let the transfer function of these two fractional discrete-time systems be given, respectively, by

(a) $H_{\mathrm{bd}}(z)=\left(\frac{1-z^{-1}}{T}\right)^{\alpha}, \quad|z|>1$

and

(b) $H_{\text {bil }}(z)=\left(\frac{2}{T} \frac{1-z^{-1}}{1+z^{-1}}\right)^{\alpha}, \quad|z|>1$.

Fractional differentiators and integrators are obtained, respectively, with $\alpha>0$ and $\alpha<0$.
The computation of the inverse $Z$-transform of (2) is simple using the binomial series expansion:

$h_{\mathrm{bd}}(n)=\frac{1}{T^{\alpha}}\left(\begin{array}{l}\alpha \\ n\end{array}\right)(-1)^{n} u_{n}=\frac{1}{T^{\alpha}} \frac{(-\alpha)_{n}}{n !} u_{n}$,

where $u_{n}$ is the discrete-time Heaviside function and $(a)_{n}=a(a+1) \ldots(a+n-1)$ is the Pochhammer symbol.

Considering (3), it can be seen that the corresponding impulse response is actually a convolution of two binomial sequences corresponding to the numerator and the denominator. It is not difficult to obtain

$h_{\mathrm{bil}}(n)\left(\frac{2}{T}\right)^{\alpha} \sum_{k=0}^{n} \frac{(-\alpha)_{k}}{k !} \frac{(-1)^{n-k}(\alpha)_{n-k}}{(n-k) !}$.

As

$n !=(-1)^{k}(-n)_{k}(n-k) ! \quad k \leqslant n$

and

$(a)_{n}=(-1)^{k}(-a-n+1)_{k}(a)_{n-k}$

we obtain

$$
\begin{aligned}
& h_{\mathrm{bil}}(n) \\
& \quad=\left(\frac{2}{T}\right)^{\alpha} \frac{(-1)^{n}(\alpha)_{n}}{n !} \sum_{k=0}^{n} \frac{(-\alpha)_{k}(-n)_{k}}{(-\alpha-n+1)_{k}} \frac{(-1)^{k}}{k !} \\
& \quad=\left(\frac{2}{T}\right)^{\alpha} \frac{(-1)^{n}(\alpha)_{n}}{n !}{ }_{2} F_{1}(-\alpha,-n ;-\alpha-n+1,-1),
\end{aligned}
$$

where ${ }_{2} F_{1}(a, b ; c,-1)$ is the Gauss hypergeometric function that, for these arguments, does not have a closed form. (Prof. Volker Strehl, in a personal communication, stated that, almost surely, ${ }_{2} F_{1}(-\alpha,-n ;-\alpha-n+1 ;-1)$ satisfies a second order recursion formula.)

\subsection{The algorithm}

Both discrete-time representations are IIR systems, but are not described by finite orders ARMA models. However, we intend to find approximations using finite orders ARMA models. There have been a lot of attempts to do it $[4-6,8]$. Here we propose a new LS identification algorithm different from that one proposed in [4].

According to our previous considerations, we assume without any loss of generality that the correct model is an $\operatorname{ARMA}(\infty, \infty)$. This is valid not only for the above referred differintegrator, but also for any other transfer function. In the following, we 
will consider only these cases. The approximation we are looking for may be stated as

$H(z)=\frac{\sum_{m=0}^{\infty} f_{m} z^{-m}}{\sum_{n=0}^{\infty} g_{n} z^{-n}} \approx \frac{\sum_{m=0}^{M} b_{m} z^{-m}}{\sum_{n=0}^{N} a_{n} z^{-n}}$.

It is not difficult to see that we are dealing with an indeterminate problem, and we are going to propose an easy way to overcome it. Let (8) be written as

$\sum_{n=0}^{N} a_{n} z^{-n} \sum_{m=0}^{\infty} f_{m} z^{-m} \approx \sum_{m=0}^{M} b_{m} z^{-m} \sum_{n=0}^{\infty} g_{n} z^{-n}$

and make an inverse Z-transform to obtain

$\sum_{n=0}^{N} a_{i} f_{n-i} \approx \sum_{i=0}^{M} b_{i} g_{n-i}, \quad n \in Z^{+}$.

This relation suggests us to define an error sequence by

$e_{n}=\sum_{n=0}^{N} a_{i} f_{n-i}-\sum_{i=0}^{M} b_{i} g_{n-i}, \quad n \in Z^{+}$.

Let us assume that we have $L$ values of the impulse response we want to model. The error energy is given by

$E=\sum_{n=0}^{L-1} e_{n}^{2}=\sum_{n=0}^{L-1}\left[\sum_{n=0}^{N} a_{i} f_{n-i}-\sum_{i=0}^{M} b_{i} g_{n-i}\right]^{2}$.

Let $a_{0}=1$. We are now going to compute the unknown parameters through the derivatives of $E$ in order to the ARMA parameters. This procedure is similar to that used in [11]. Therefore, we obtain the following sets of normal equations:

$$
\begin{aligned}
& \sum_{n=0}^{L-1}\left[\sum_{i=0}^{N} a_{i} f_{n-i} f_{n-k}-\sum_{i=0}^{M} b_{i} g_{n-i} f_{n-k}\right]=0, \\
& \quad k=1,2, \ldots, N
\end{aligned}
$$

and

$$
\begin{aligned}
& \sum_{n=0}^{L-1}\left[-\sum_{i=0}^{N} a_{i} f_{n-i} g_{n-k}+\sum_{i=0}^{M} b_{i} g_{n-i} g_{n-k}\right]=0, \\
& \quad k=0,1,2, \ldots, M .
\end{aligned}
$$

Introducing the covariance matrices:

$$
\begin{aligned}
& R_{f f}(k, i)=\sum_{n=0}^{L-1} f_{n-i} f_{n-k}, \\
& R_{g f}(k, i)=\sum_{n=0}^{L-1} g_{n-i} f_{n-k},
\end{aligned}
$$

$R_{g g}(k, i)=\sum_{n=0}^{L-1} g_{n-i} g_{n-k}$

we can rewrite (13) and (14) as

$\sum_{i=0}^{N} a_{i} R_{f f}(k, i)-\sum_{i=0}^{M} b_{i} R_{g f}(k, i)=0, \quad k=1,2, \ldots, N$

and

$$
\begin{aligned}
& \sum_{i=0}^{N} a_{i} R_{f g}(k, i)-\sum_{i=0}^{M} b_{i} R_{g g}(k, i)=0, \\
& \quad k=0,1,2, \ldots, N .
\end{aligned}
$$

If we know all the impulse response values or the theoretical expressions, we can compute the correlations

$$
R_{f f}(k-i)=\sum_{n=0}^{\infty} f_{n} f_{n+i-k}
$$

$R_{g f}(k-i)=\sum_{n=0}^{\infty} g_{n} f_{n+i-k}$,

$R_{g g}(k-i)=\sum_{n=0}^{\infty} g_{n} g_{n+i-k}$

that allow us to rewrite (18) and (19) as

$$
\begin{aligned}
& \sum_{i=0}^{N} a_{i} R_{f f}(k-i)-\sum_{i=0}^{M} b_{i} R_{g f}(k-i)=0, \\
& \quad k=1,2, \ldots, N
\end{aligned}
$$

and

$$
\begin{aligned}
& \sum_{i=0}^{N} a_{i} R_{f g}(k-i)-\sum_{i=0}^{M} b_{i} R_{g g}(k-i)=0, \\
& \quad k=0,1,2, \ldots, N .
\end{aligned}
$$

With this formulation all the involved matrices are Toeplitz matrices. However, we will maintain the notation introduced in (18) and (19). We can join all the matrices in only one. Introduce the matrices $\boldsymbol{\Phi}[(N+1) \times(N+1)], \boldsymbol{\Psi}[(M+1) \times(N+1)] \quad$ and $\boldsymbol{\Theta}[(M+1) \times(M+1)]$ that are easily identified and a $(N+M+2)$ length vector $\mathbf{w}=\left[1 a_{1} a_{2} \ldots\right.$ 
$\left.a_{N} b_{0} b_{1} b_{2} \ldots b_{M}\right]^{\mathrm{T}}$. We join (18) and (19) into the following system of equations:

$$
\left[\begin{array}{cc}
\boldsymbol{\Phi} & -\boldsymbol{\Psi} \\
\boldsymbol{\Psi}^{\mathbf{T}} & -\boldsymbol{\Theta}
\end{array}\right]\left[\begin{array}{c}
1 \\
a_{1} \\
\vdots \\
a_{N} \\
b_{0} \\
\vdots \\
b_{M}
\end{array}\right]=\left[\begin{array}{c}
E_{\min } \\
0 \\
\\
\cdot \\
\cdot \\
0
\end{array}\right]
$$

The solution is readily obtained by inverting the matrix. We only have to obtain the first column of the inverse and normalize the first coefficient. The normalizing constant is equal to $E_{\min }$. The partitioned inverses formula can be used here [12]. To our needs it is enough to say that the first column of the following matrix is the solution (non-normalized) for our problem:

$$
E_{\min }\left[\begin{array}{c}
1 \\
a_{1} \\
\vdots \\
a_{N} \\
b_{0} \\
\vdots \\
b_{M}
\end{array}\right]=\left[\begin{array}{l}
\left(\boldsymbol{\Phi}-\boldsymbol{\Psi} \boldsymbol{\Theta}^{-1} \boldsymbol{\Psi}^{\mathbf{T}}\right)^{-1} \\
-\boldsymbol{\Psi} \boldsymbol{\Theta}^{-1} \boldsymbol{\Psi}^{\mathbf{T}}\left(\boldsymbol{\Phi}-\boldsymbol{\Psi} \boldsymbol{\Theta}^{-1} \boldsymbol{\Psi}^{\mathbf{T}}\right)^{-1}
\end{array}\right]\left[\begin{array}{c}
1 \\
0 \\
\cdot \\
\cdot \\
\\
0
\end{array}\right]
$$

provided that either $\left(\boldsymbol{\Phi}-\boldsymbol{\Psi} \boldsymbol{\Theta}^{-1} \boldsymbol{\Psi}^{\mathbf{T}}\right)$ or $\boldsymbol{\Theta}$ are regular. If this is not the case, we can use

$$
E_{\min }\left[\begin{array}{c}
1 \\
a_{1} \\
\vdots \\
a_{N} \\
b_{0} \\
\vdots \\
b_{M}
\end{array}\right]
$$

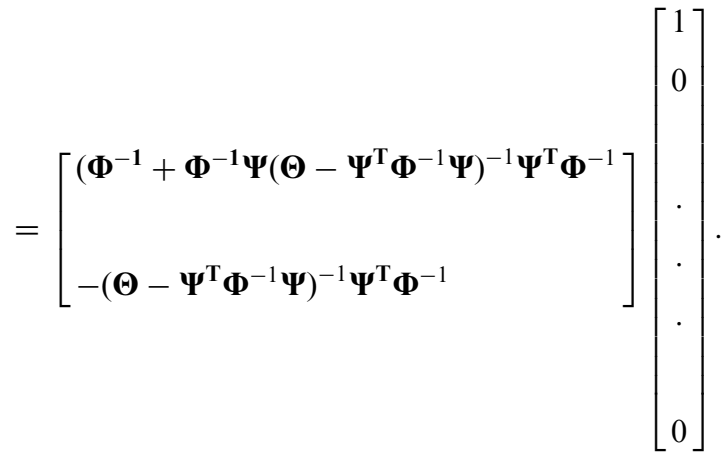

We remark here that $a_{0}=1$ and thus

$$
\begin{aligned}
& {\left[\sum_{i=1}^{N} a_{i} R_{f f}(k-i)-\sum_{i=0}^{M} b_{i} R_{g f}(k-i)\right]=-R_{f f}(k),} \\
& \quad k=1,2, \ldots, N
\end{aligned}
$$

and

$$
\begin{aligned}
& {\left[\sum_{i=1}^{N} a_{i} R_{f g}(k-i)-\sum_{i=0}^{M} b_{i} R_{g g}(k-i)\right]=-R_{f g}(k),} \\
& \quad k=0,1,2, \ldots, M .
\end{aligned}
$$

This may be interesting because it allows a reduction in the dimensions of matrices $\boldsymbol{\Phi}$ and $\boldsymbol{\Psi}$. It is not difficult to obtain similar matrices to those in (25)-(27).

\subsection{Applications}

\subsubsection{Difference}

We are going to use the previous algorithm to compute the approximation to the differintegrator using the backward difference. Assume that $\alpha>0$. In this case and referring to the notation used in the previous section, we have

$f_{n}=\frac{1}{T^{\alpha}} \frac{(-\alpha)_{n}}{n !} u_{n}$

and

$g_{n}=\delta_{n}$.

Let the corresponding correlations be computed:

$$
\begin{aligned}
R_{f f}(n) & =\frac{1}{T^{2 \alpha}} \sum_{k=0}^{\infty}\left(\begin{array}{c}
\alpha \\
k
\end{array}\right)(-1)^{k}\left(\begin{array}{c}
\alpha \\
k+n
\end{array}\right)(-1)^{k+n} \\
& =\frac{1}{T^{2 \alpha}} \sum_{k=0}^{\infty} \frac{(-\alpha)_{k}}{k !} \frac{(-\alpha)_{k+n}}{(k+n) !}
\end{aligned}
$$


As

$(n+k) !=(n+1)_{k} n !$

and

$(\alpha)_{n+k}=(\alpha)_{n}(\alpha+n)_{k}$,

$$
\begin{aligned}
& \frac{1}{T^{2 \alpha}} \frac{(-\alpha)_{n}}{n !} \sum_{k=0}^{\infty} \frac{(-\alpha)_{k}}{k !} \frac{(-\alpha+n)_{k}}{(n+1) !} \\
& \frac{1}{T^{2 \alpha}} \frac{(-\alpha)_{n}}{n !}{ }_{2} F_{1}(-\alpha,-\alpha+n ; n+1 ; 1) .
\end{aligned}
$$

Using the Gauss formula

${ }_{2} F_{1}(a, b ; c ; 1)=\frac{\Gamma(c) \Gamma(c-a-b)}{\Gamma(c-a) \Gamma(c-b)} \quad c-a-b>0$

we obtain

$$
R_{f f}(k)=\frac{1}{T^{2 \alpha}}(-1)^{k} \frac{\Gamma(1+2 \alpha)}{\Gamma(\alpha+k+1) \Gamma(\alpha-k+1)}
$$

meaning that the $\Phi$ matrix is a symmetric Toeplitz matrix. Obviously

$R_{g f}(n)=\frac{1}{T^{\alpha}}\left(\begin{array}{l}\alpha \\ n\end{array}\right)(-1)^{n} u_{n}$.

Then, $\Psi$ is a non-symmetric Toeplitz matrix. If $\alpha<0$, it is enough to interchange $f_{n}$ with $g_{n}$.

\subsubsection{Bilinear}

Assume again that $\alpha>0$. For the $f_{n}$ sequence, we use the above expression (30). For $g_{n}$ we have

$g_{n}=\left(\begin{array}{l}\alpha \\ n\end{array}\right) u_{n}$

The corresponding autocorrelation is

$R_{g g}(k)=\frac{\Gamma(1+2 \alpha)}{\Gamma(\alpha+k+1) \Gamma(\alpha-k+1)}$

leading again to a symmetric Toeplitz matrix. The cross-correlation is given for $n>0$ and $\alpha<1$

$$
\begin{aligned}
R_{f g}(n) & =\frac{1}{T^{\alpha}} \sum_{k=0}^{\infty}\left(\begin{array}{l}
\alpha \\
k
\end{array}\right)(-1)^{k}\left(\begin{array}{c}
\alpha \\
k+n
\end{array}\right) \\
& =\frac{1}{T^{\alpha}} \sum_{k=0}^{\infty} \frac{(-\alpha) k}{k !} \frac{(-\alpha)_{k+n}}{(k+n) !} \\
& =\frac{1}{T^{\alpha}} \frac{(-\alpha)_{n}}{n !} \sum_{k=0}^{\infty} \frac{(-\alpha)_{k}(-\alpha+n)_{k}}{(1+n)_{k}} \\
& =\frac{1}{T^{\alpha}} \frac{(-\alpha)_{n}}{n !}{ }_{2} F_{1}(-\alpha+n,-\alpha ; n+1 ;-1) .
\end{aligned}
$$

From Kummer's formula [13]

$$
\begin{aligned}
{ }_{2} F_{1}(a, b ; a-b+1 ;-1)= & \frac{\Gamma(1+a-b) \Gamma(1+a / 2)}{\Gamma(1+a / 2-b) \Gamma(a+1)}, \\
& b<1 \text { and a - b non-negative integer }
\end{aligned}
$$

we obtain

$$
\begin{aligned}
R_{f g}(n) & =\frac{1}{T^{\alpha}} \frac{(-\alpha)_{n}}{n !} \frac{\Gamma(1-\alpha+n) \Gamma(1-\alpha / 2+n / 2)}{\Gamma(1+\alpha / 2+n / 2) \Gamma(-\alpha+n+1)} \\
& =\frac{1}{T^{\alpha}} \frac{(-1)^{n}}{\Gamma(-\alpha)(-\alpha+n)} \frac{\Gamma(1-\alpha / 2+n / 2)}{\Gamma(1+\alpha / 2+n / 2)} .
\end{aligned}
$$

If $n<0$

$R_{f g}(n)=(-1)^{n} R_{f g}(-n)$

leading to a non-symmetric Toeplitz matrix. In this case and if $N=M$, we have

$b_{i}=(-1)^{i} a_{i}$

suggesting the usage of an $\operatorname{ARMA}(N, N)$.

\section{Comparisons}

In this section we are going to use the above algorithm to compute approximations for the differintegrator. However, only the results for the differentiator $(\alpha>0)$ are presented since, for practical purposes, the integrator does not constitute a different case study. This leads us to consider fractional orders $\alpha \in[-0.5,0.5]$. For comparisons, we will use the results presented in $[3,4]$ whenever feasible. ${ }^{1}$

In order to get some insight into the order of the ARMA models, experiments with $\operatorname{ARMA}(n, m), n=$ $1, \ldots, N$ and $m=0, \ldots, n$ were performed. Figs. 1 and 2 depict the behavior of $E_{\text {min }}$, for the backwards difference and bilinear, respectively, as by Eq. (26) as a function of $(n, m)$. It is interesting to note that in both cases, the local minima of $E_{\min }$ occur when $n=m$, that is, the number of poles equals the number of zeros. Also note that maxima correspond to $\operatorname{AR}(N)$ models.

Further simulations were carried out for both the backward differences and bilinear $\operatorname{MA}(M)$ models. Fig. 3 (backward differences) and Fig. 4 (bilinear) depict $E_{\min }$ as a function of model order (left graph) and the final zero map (right graph).

\footnotetext{
${ }^{1}$ Although in [3] we where able to find an $\operatorname{ARMA}(9,9)$, using the LS approach, an $\operatorname{ARMA}(N, N)$ was unstable for $N>6$, both for $\alpha=0.5$ and using the backward differences.
} 


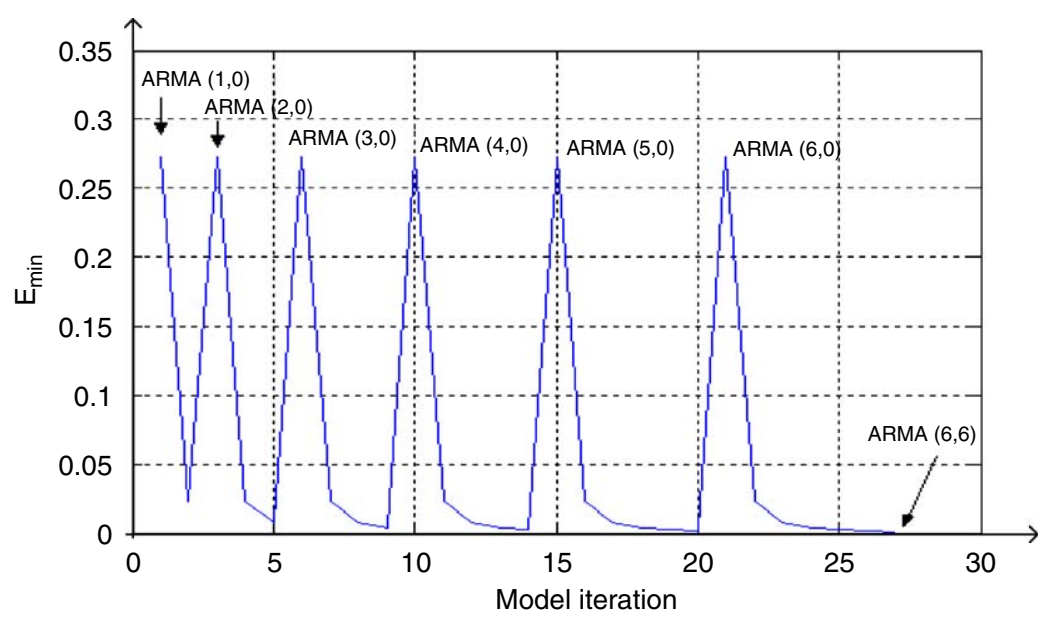

Fig. 1. $E_{\min }$ as a function of model order for $\alpha=0.5$ and backward differences. Local minima correspond to $\operatorname{ARMA}(n, n)$ and the last sample being for ARMA(6,6).

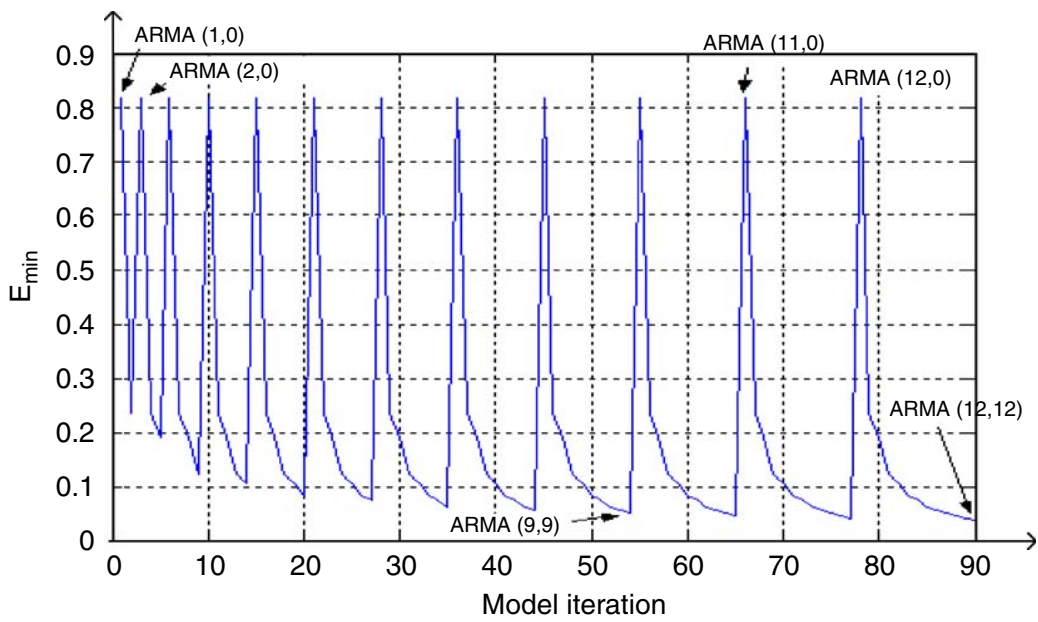

Fig. 2. $E_{\min }$ as a function of model order for $\alpha=0.5$ and bilinear. Local minima corresponds to ARMA $(n, n)$ and the last sample being for $\operatorname{ARMA}(12,12)$.

Based on the $E_{\min }$ behavior as a function of the order of the models, all the remaining experiments comprise only $\operatorname{ARMA}(N, N)$ models.

As Figs. 5 and 6 depict, there is no substantial or significant difference between the algorithm proposed in this work, LS, and that presented in [3].

In the LS case, higher ARMA orders can be thought but, for comparison purposes with the previous algorithm, we limited them to $(9,9)$. In fact, using the LS approach, and for $\alpha=0.5$, we were able to estimate an $\operatorname{ARMA}(12,12)$, depicted in Fig. 7. It should be pointed out that, for this same value of $\alpha=0.5$, in [3] instability problems occurred for $(N, N)>9$.
Changing the value of $\alpha$ to 0.1 leads us to Fig. 8 (backward differences) and Fig. 9 (bilinear). These approximations are summarized in Table 1.

Values of $|\alpha|>0.5$ are not interesting to present since they can be trivially reduced to a "new" fractional order $\alpha^{*}=(1-\alpha)$ such that $\alpha^{*} \in[0,0.5]$ by including either an integrator or a differenciator that results in an extra $\left(1-z^{-1}\right)^{-1}$ or $\left(1-z^{-1}\right)$ factor, respectively. This extra factor can have adverse effects in control applications, specially if it results as an integrator. In this case, $\alpha$ should be restricted to $[0,1]$, instead.

Unfortunately, no definitive assertions can be made on the order of the ARMA models since no 

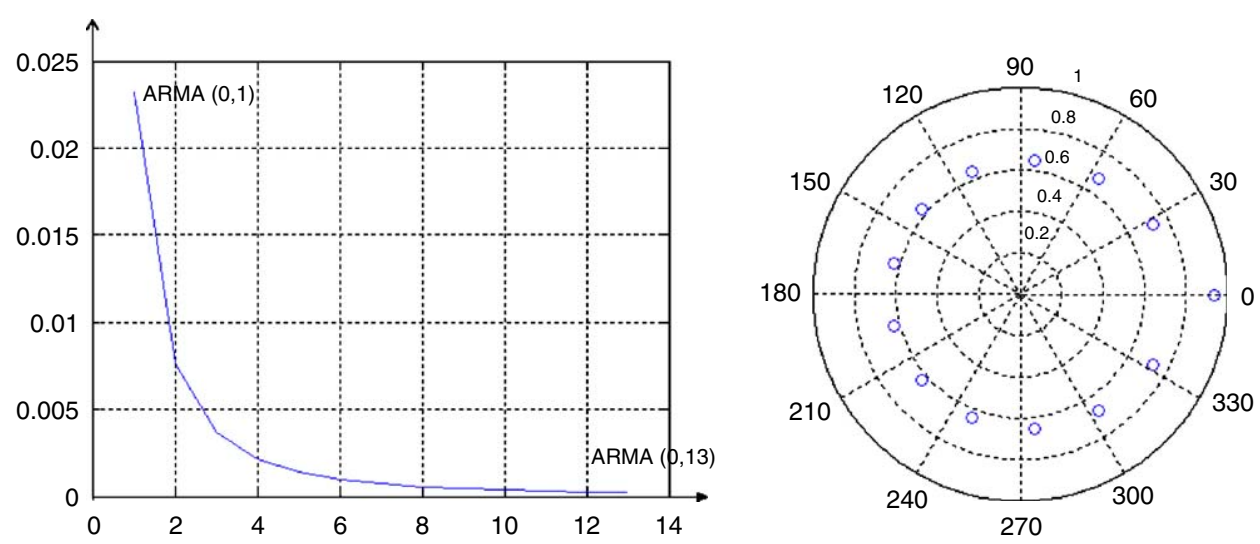

Fig. 3. $E_{\min }$ (left plot) for $\operatorname{ARMA}(0, m), m=1, \ldots, 13$ and zeros on the $z$-plane for $\operatorname{ARMA}(0,13)$ (right plot) both for $\alpha=0.5$ and backward differences.
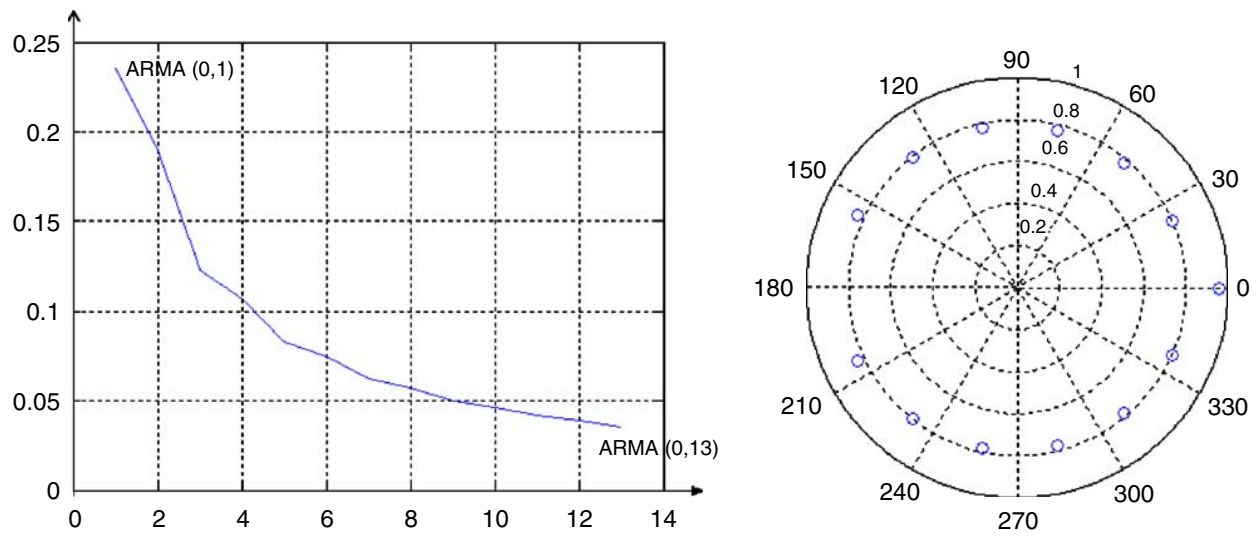

Fig. 4. $E_{\min }$ (left plot) for $\operatorname{ARMA}(0, m), m=1, \ldots, 13$ and zeros on the $z$-plane for $\operatorname{ARMA}(0,13)$ (right plot) both for $\alpha=0.5$ and bilinear.
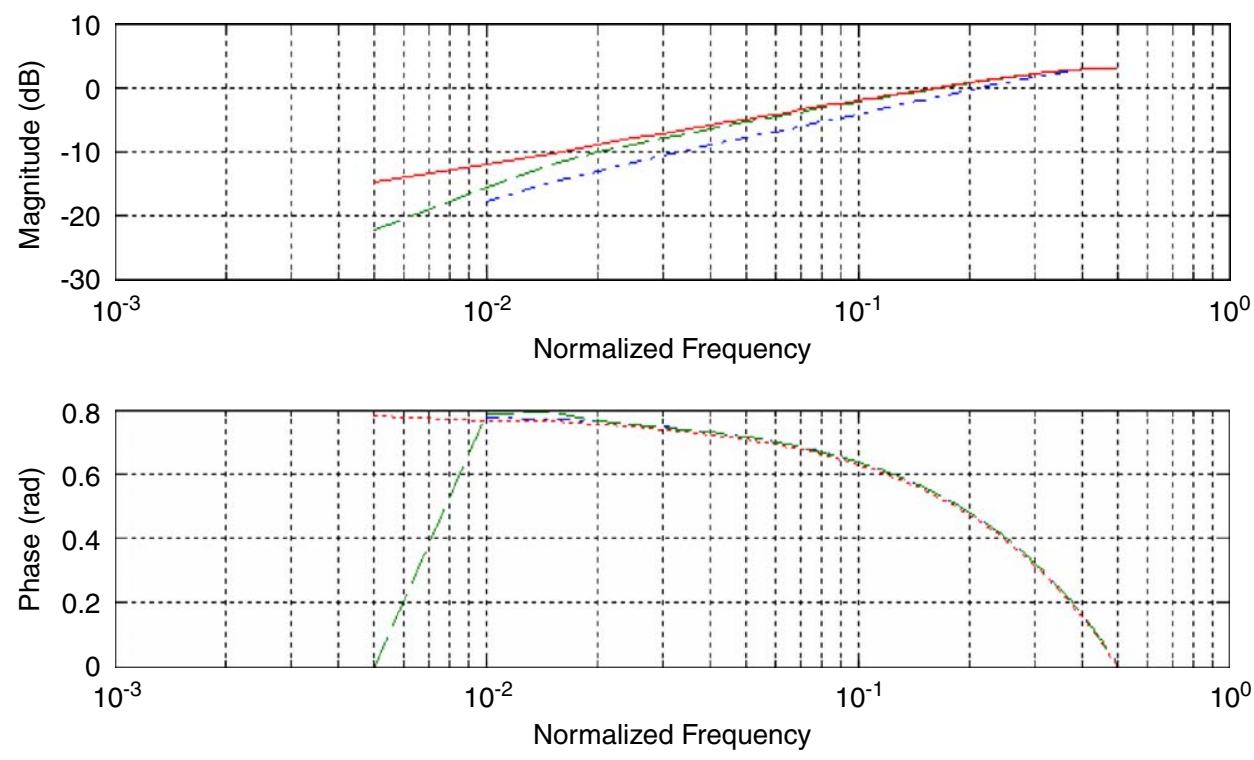

Fig. 5. ARMA $(6,6)$ amplitude (top, in $\mathrm{dB}$ ) and phase (bottom, in radians) frequency response plots for the backward difference, with $\alpha=0.5$. LS approach is the solid line, the exact model spectrum is the dash-dotted line, while as in [2] is the dashed line. 

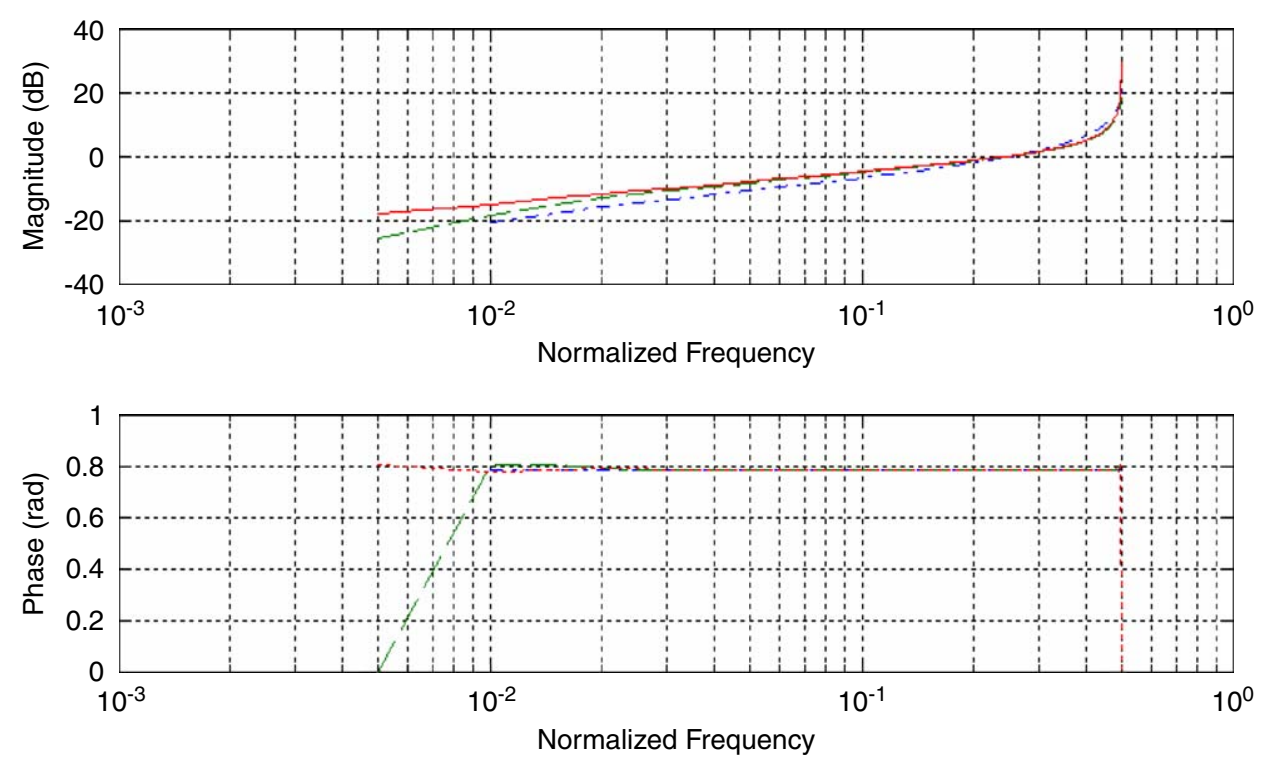

Fig. 6. ARMA $(9,9)$ amplitude (top, in $\mathrm{dB}$ ) and phase (bottom, in radians) frequency response plots for the bilinear, with $\alpha=0.5$. LS approach is the solid line, the exact model spectrum is the dash-dotted line, while as in [7] is the dashed line.
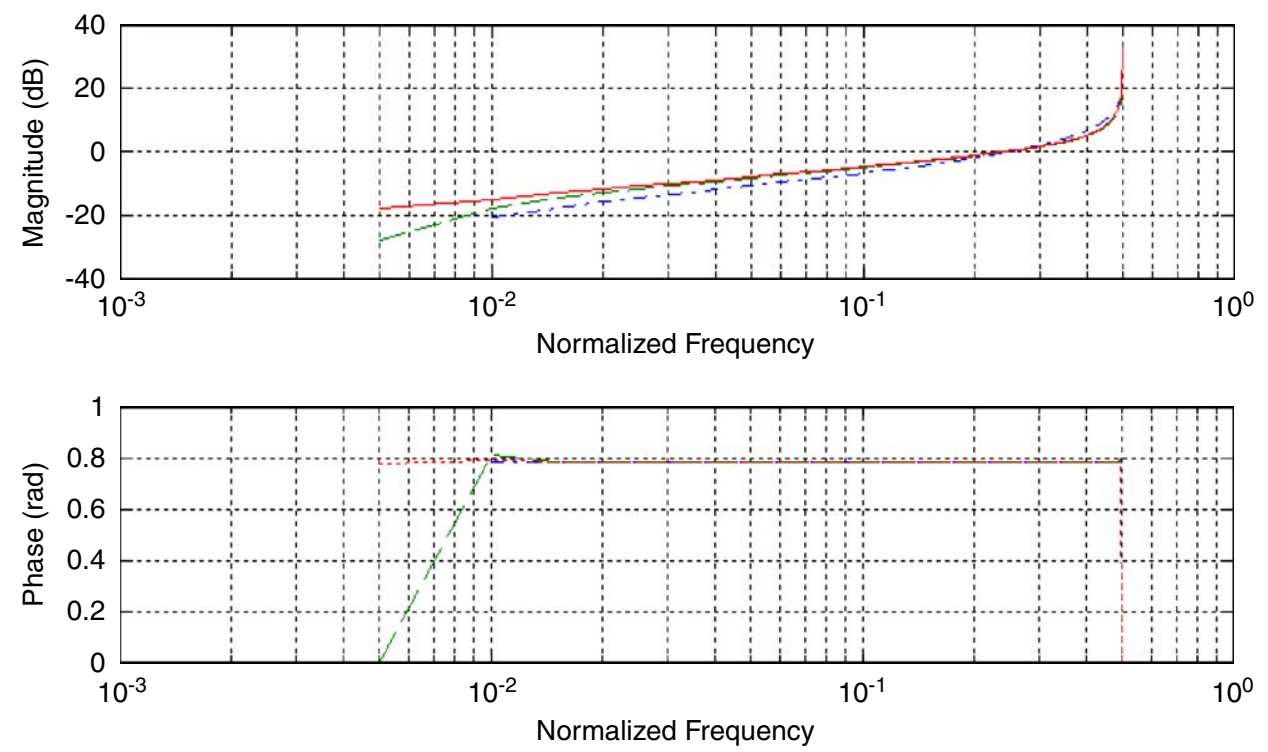

Fig. 7. ARMA(12,12) amplitude (top, in $\mathrm{dB}$ ) and phase (bottom, in radians) frequency response plots for the bilinear, with $\alpha=0.5$. LS approach is the solid line and the exact model spectrum is the dash-dotted line.

further evidence was drawn from the simulations we performed. However, the bilinear seems to be more stable than the backward differences, and this behavior appears to be valid for values of $\alpha \in\{0.1,0.5,0.8\}$. As a final note, and for the presented examples (regardless the value of $\alpha$ and the chosen approximation), all the poles and zeros were real-valued as long as models remain $\operatorname{ARMA}(N, N)$. 

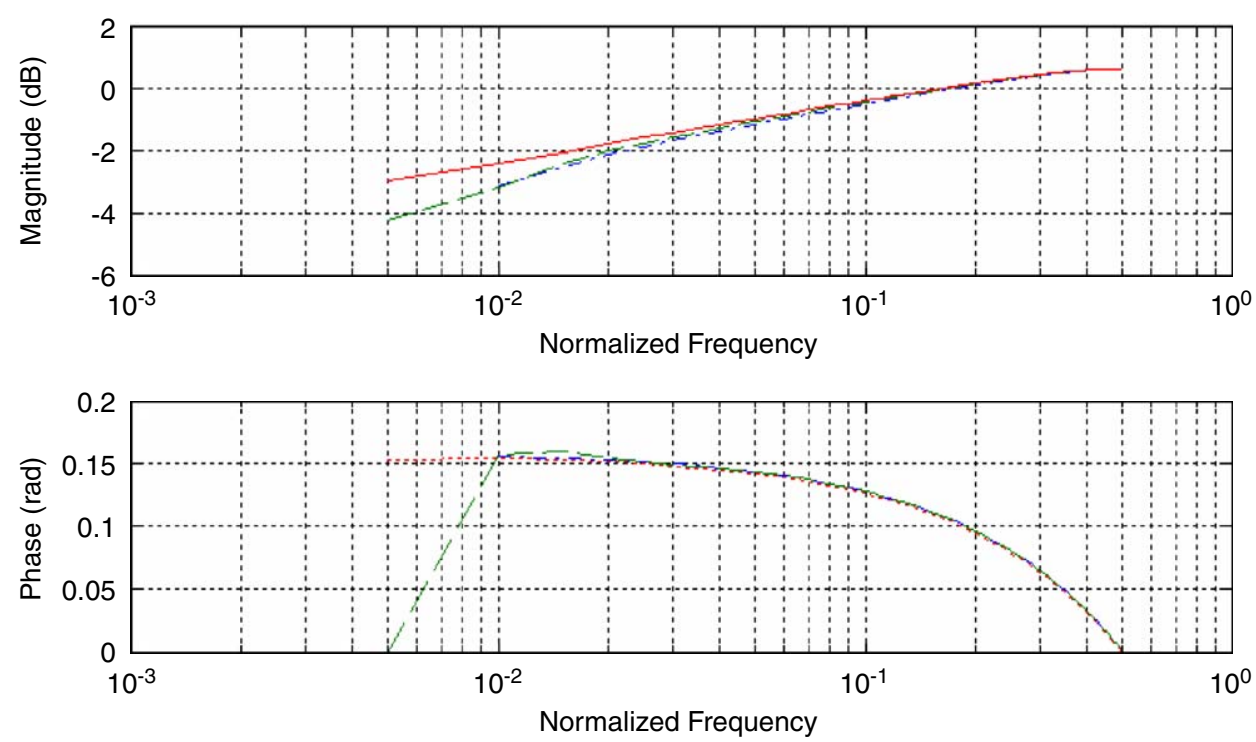

Fig. 8. ARMA $(6,6)$ amplitude (top, in $\mathrm{dB}$ ) and phase (bottom, in radians) frequency response plots for the backward difference, with $\alpha=0.1$. LS approach is the solid line, the exact model spectrum is the dash-dotted line, while as in [2] is the dashed line.
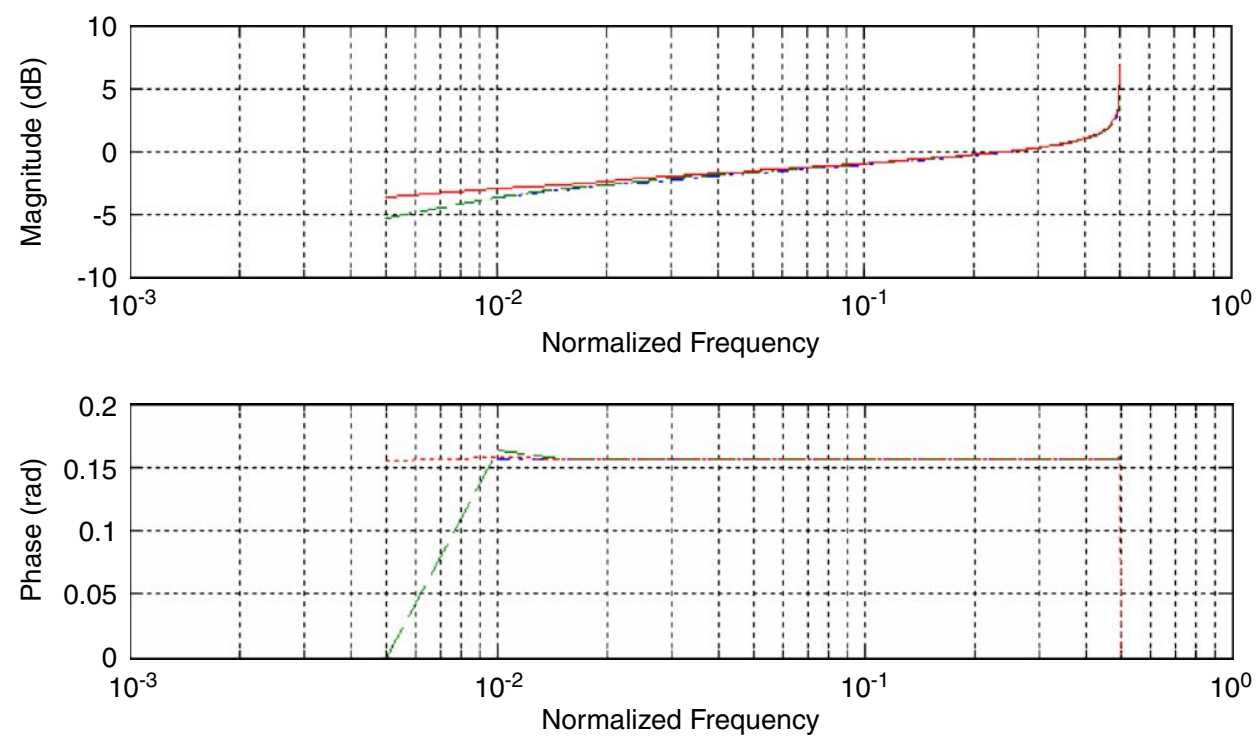

Fig. 9. ARMA(12,12) amplitude (top, in $\mathrm{dB}$ ) and phase (bottom, in radians) frequency response plots for the bilinear, with $\alpha=0.1$. LS approach is the dotted line and the exact model spectrum is the dash-dotted line.

\section{Conclusions}

We proposed here a new LS algorithm for pole-zero modeling of fractional linear systems. This is based on an error power minimization relatively to the ARMA models that leads to a set of normal equations. We applied to two well-known situations consisting of the difference and bilinear transformations that are suitable for exact autocorrelation computation. Some illustrating examples were presented showing that $\operatorname{ARMA}(N, N)$ are suitable models. 
Table 1

Selected transfer functions for the LS approach

\begin{tabular}{|c|c|c|c|c|}
\hline$\alpha$ & ARMA & Model & AR coefficients & MA coefficients \\
\hline 0.1 & $(6,6)$ & Backward & $\begin{array}{l}1.0000 ;-3.4875 ; 4.7052 ;-3.0595 \\
0.9645 ;-0.1269 ; 0.0041\end{array}$ & $\begin{array}{l}1.0000 ;-3.5875 ; 5.0090 ;-3.4016 \\
1.1375 ;-0.1638 ; 0.0064\end{array}$ \\
\hline 0.1 & $(12,12)$ & Bilinear & $\begin{array}{l}1.0000 ; 0.0997 ;-3.3770 ;-0.3037 \\
4.3668 ; 0.3438 ;-2.6756-0.1753 ; \\
0.7699 ; 0.0381 ;-0.0857 ;-0.0025 \\
0.0017\end{array}$ & $\begin{array}{l}1.0000 ; 0.1003 ;-3.3770 ; 0.30574 .3666 ; \\
-0.3460 ;-2.6755 ; 0.1765 ; 0.7699 ; \\
-0.0383 ;-0.0857 ; 0.0025 ; 0.0017\end{array}$ \\
\hline 0.5 & $(6,6)$ & Backward & $\begin{array}{l}1.0000 ;-3.2112 ; 3.9246 ;-2.2548 \\
0.6019 ;-0.0612 ; 0.0011\end{array}$ & $\begin{array}{l}1.0000 ;-3.7112 ; 5.4052 ;-3.8782 \\
1.4004 ;-0.2274 ; 0.0113\end{array}$ \\
\hline 0.5 & $(9,9)$ & Bilinear & $\begin{array}{l}1.0000 ; 0.5000 ;-2.3019-1.0260 \\
1.7917 ; 0.6706 ;-0.5282 ;-0.1450 \\
0.0437 ; 0.0052\end{array}$ & $\begin{array}{l}1.0000 ;-0.5000 ;-2.3019 ; 1.0260 ; \\
1.7917 ;-0.6706 ;-0.5282 ; 0.1450 ; \\
0.0437-0.0052\end{array}$ \\
\hline 0.5 & $(12,12)$ & Bilinear & $\begin{array}{l}1.0000 ; 0.5001 ;-3.1883 ;-1.4696 ; \\
3.8645 ; 1.5967 ;-2.1989 ;-0.7768 \\
0.5801 ; 0.1596 ;-0.0581 ;-0.0097 \\
0.0010\end{array}$ & $\begin{array}{l}1.0000 ;-0.4999 ;-3.1884 ; 1.4688 \\
3.8648 ;-1.5959 ;-2.1992 ; 0.7764 ; \\
0.5802 ;-0.1595 ;-0.0581 ; 0.0097 \\
0.0010\end{array}$ \\
\hline
\end{tabular}

\section{References}

[1] M.D. Ortigueira, ARMA realization from the reflection coefficient sequence, Signal Processing 32 (3) (June 1993).

[2] M.D. Ortigueira, F.V.C. Coito, From differences to differintegrations, Fractional Calculus Appl. Anal. 7 (4) (2004).

[3] M.D. Ortigueira, A.J.S. Serralheiro, Pseudo-fractional ARMA modelling using a double Levinson recursion, IEE Proc. Control Theory Appl., accepted for publication.

[4] R.S. Barbosa, J.A. Tenreiro Machado, I.M. Ferreira, Leastsquares design of digital fractional-order operators, FDA'2004 First IFAC Workshop on Fractional Differentiation and Its Applications, Bordeaux, France, July 19-21, 2004.

[5] R.S. Barbosa, J.A. Tenreiro Machado, I.M. Ferreira, Pole-zero approximations of digital fractional-order integrators and differentiators using signal modeling techniques, Proceedings of the 16th IFAC World Congress, Prague, Czech Republic, July 2005.

[6] Y.Q. Chen, B.M. Vinagre, A new IIR-type digital fractional order differentiator, Signal Processing 83 (2003) 2359-2365.
[7] P. Ostalczyk, Fundamental properties of the fractional-order discrete-time integrator, Signal Processing 83 (2003) 2367-2376.

[8] J.A. Tenreiro Machado, Discrete-time fractional-order controllers, Fractional Calculus Appl. Anal. J. 1 (2001) 47-66.

[9] B.M. Vinagre, I. Podlubny, A. Hernandez, V. Feliu, Some approximations of fractional order operators used in control theory and applications, Fractional Calculus Appl. Anal. 3 (3) (2000) 231-248.

[10] M.D. Ortigueira, J.A. Tenreiro-Machado, J. Sá da Costa, Which differintegration? IEE Proc. Vision Image Signal Process 152 (6) (2005) 846-850.

[11] K. Steiglitz, On the simultaneous estimation of poles and zeros in speech analysis, IEEE Trans. Acoust. Speech Signal Process. ASSP-25 (3) (June 1977).

[12] H. Lütkepohl, Handbook of Matrices, Wiley, New York, 1996.

[13] P. Henrici, Applied and Computational Complex Analysis, vol. 2, Wiley, New York, 1991, pp. 389-319. 